\title{
Mucosal delivery of human papillomavirus pseudovirus-encapsidated plasmids improves the potency of DNA vaccination
}

\author{
BS Graham ${ }^{1,4}$, RC Kines ${ }^{2,4}$, KS Corbett ${ }^{1}$, J Nicewonger ${ }^{1}$, TR Johnson ${ }^{1}$, M Chen ${ }^{1}$, D LaVigne ${ }^{1}$, \\ JN Roberts ${ }^{3}$, N Cuburu ${ }^{2}$, JT Schiller ${ }^{2}$ and CB Buck ${ }^{2}$
}

\begin{abstract}
Mucosal immunization may be important for protection against pathogens whose transmission and pathogenesis target the mucosal tissue. The capsid proteins of human papillomavirus (HPV) confer tropism for the basal epithelium and can encapsidate DNA during self-assembly to form pseudovirions (PsVs). Therefore, we produced mucosal vaccine vectors by HPV PsV encapsidation of DNA plasmids expressing an experimental antigen derived from the M and M2 proteins of respiratory syncytial virus (RSV). Intravaginal (IVag) delivery elicited local and systemic M/M2-specific CD8+ T-cell and antibody responses in mice that were comparable to an $\sim 10,000$-fold higher dose of naked DNA. A single HPV PsV IVag immunization primed for M/M2-specific-lgA in nasal and vaginal secretions. Based on light emission and immunofluorescent microscopy, immunization with HPV PsV-encapsidated luciferase- and red fluorescent protein (RFP)-expressing plasmids resulted in transient antigen expression ( $<5$ days), which was restricted to the vaginal epithelium. HPV PsV encapsidation of plasmid DNA is a novel strategy for mucosal immunization that could provide new vaccine options for selected mucosal pathogens.
\end{abstract}

\section{INTRODUCTION}

Vaccination by gene delivery is an important immunization strategy for viral diseases. Expression of viral proteins from the host cell can authentically mimic the native structure and antigenicity of viral proteins and provide a platform for the induction of both humoral and cellular immunity. There are many potential gene-based vectors that can deliver the genes encoding vaccine antigens, and each has a unique set of properties that determine its usage. These include manufacturing feasibility, cost, stability, ease of storage and administration, replication competence, vector-specific toxicity, pre-existing vector immunity in target host, immune competition between vector antigens and vaccine antigen(s), nuclear or cytoplasmic transcription of transgene, tissue tropism, host range, and potential routes of administration. Naked DNA is the simplest platform for gene delivery. It avoids vector-related concerns, is easy to make, and temperature-stable. The major detraction of DNA is that it does not efficiently enter cells and has low potency relative to the number of gene copies per dose.
Immunogenicity has been improved by using various formulations, vehicles, and devices that improve gene expression after parenteral administration, but methods to immunize mucosal surfaces have not been advanced. ${ }^{1}$

Vaccines for some viral pathogens that typically invade mucosal surfaces such as human immunodeficiency virus type 1 (HIV-1), herpes simplex virus, or respiratory syncytial virus (RSV), have been difficult to develop in part because of the potential need for mucosal immunity. Although passively acquired antibodies can protect against these types of diseases, ${ }^{2-4}$ induction of immune responses locally at the site of infection may provide an advantage. Replication-competent viral vectors have typically been more immunogenic when delivered to mucosal surfaces than inactivated virus or replication-defective vaccine vectors, but safety concerns involving biodistribution, integration, and virulence complicate their development.

Despite the immunogenicity of replication-defective genebased vaccine vectors administered parenterally, delivery systems specifically designed for induction of mucosal immunity have

\footnotetext{
${ }^{1}$ Viral Pathogenesis Laboratory, Vaccine Research Center, National Institute of Allergy and Infectious Diseases, Bethesda, Maryland, USA. ${ }^{2}$ Laboratory of Cellular Oncology, National Cancer Institute, National Institutes of Health, Bethesda, Maryland, USA. ${ }^{3}$ Food and Drug Administration, Bethesda, Maryland, USA. ${ }^{4}$ These authors contributed equally to this work. Correspondence: BS Graham or RC Kines (bgraham@nih.gov) 
been slow to advance. Only recombinant canarypox expressing HIV-1 and rabies virus antigens delivered by multiple mucosal routes, and nasal delivery of replication-defective recombinant adenovirus vector expressing influenza antigen, have advanced to clinical trials. ${ }^{5,6}$ Identifying approaches that effectively deliver replication-defective gene-based vaccine vectors to mucosal surfaces would provide an important option for development of future vaccines.

Sexually transmitted human papillomavirus (HPV) types are prototypic mucosal pathogens that infect the stratified squamous epithelium, particularly when microtrauma provides access to the basal epithelial layer. ${ }^{7}$ The papillomavirus major capsid protein, L1, can self-assemble into virus-like particles (VLPs) with or without the minor capsid protein, $\mathrm{L} 2 .^{8-11}$ HPV L1 VLPs are able to bind and activate dendritic cells in vitro. ${ }^{12-14}$ Furthermore, parenteral immunization with HPV L1 VLPs induces T-cell responses, ${ }^{15}$ and type-specific neutralizing antibodies, and is the basis for licensed vaccines. ${ }^{16,17}$

HPV capsids can pseudotype the $\sim 8 \mathrm{~kb}$ genome of other papillomaviruses. L1 and L2 produced by Semliki Forest virus ${ }^{18}$ or recombinant vaccinia ${ }^{19}$ can encapsidate episomal genomes of alternative papillomavirus serotypes. HPV capsids have also been used as a carrier for plasmid DNA by using a cell-free system in which VLPs are disassembled and reassembled in the presence of plasmid DNA, ${ }^{20,21}$ or by direct interaction of the assembled VLPs with the DNA. ${ }^{22}$ A more efficient strategy for producing high-titer L1-L2 HPV particles encapsidating DNA plasmids in human cell lines (HPV pseudovirions, PsVs) involves co-transfecting the L1 and L2 genes on a large plasmid $>8 \mathrm{~kb}$ with a smaller plasmid $(<8 \mathrm{~kb}$ ) expressing the genes of interest. In this setting, the reporter plasmid is preferentially packaged, together with cellular histone proteins, within the self-assembled recombinant capsid. ${ }^{23,24}$

The immunogenicity of antigens expressed from HPV PsVdelivered plasmids has been examined using particles composed of L1 only, and produced by the cell-free disassembly/reassembly method. ${ }^{21,25-28}$ Parenteral and oral administration of these pseudovirions generated detectable CTL and/or antibody responses to genes expressing HIV-1 Gag, LCMV gp33, and CEA. Recently, a potential strategy for IVag HPV-PsV-mediated gene delivery was described. ${ }^{29,30}$ Although the intact cervicovaginal tract was highly resistant to HPV PsV infection, if the epithelium was disrupted physically by a cytobrush or chemically with nonoxynol-9, gene transfer and local reporter protein expression was observed.

In this study, we explore the immunogenicity of HPV-PsVencapsidated plasmid DNA expressing a well-characterized RSV model antigen delivered to the vaginal epithelium. In particular, we compared the immunogenicity of HPV-PsV delivery of encapsidated DNA with immunization with naked DNA alone. We find that HPV PsV-mediated delivery-to-the vaginal epithelium increases the potency of plasmid DNA for eliciting CD8 + T-cell responses, increases the induction of antibody to the DNA-expressed antigen, and targets the epithelium of the vaginal mucosa resulting in a discrete period of antigen exposure. HPV PsV encapsidation facilitates entry of plasmid DNA into host cells to complement the other exceptional qualities of DNA as a gene delivery vehicle, and provides a technology with the potential for mucosal immunization.

\section{RESULTS \\ Characteristics of the HPV-encapsidated M-M2-expressing DNA}

Genes encoding the M/M2 fusion proteins were effectively expressed in 293T cells transduced with HPV-encapsidated plasmids as shown by western blot (Figure 1a). The standard dose of $5 \times 10^{7} \mathrm{IU}$ of HPV PsV-M/M2 contains about $5 \mathrm{ng}$ of reporter plasmid DNA as determined by agarose gel electrophoresis (http://home.ccr.cancer.gov/lco/encapsidateddnaanalysis.htm) (data not shown). Western blot analysis using M- and M2-specific monoclonal antibodies did not detect M/M2 protein contamination in a standard dose of the HPV PsV preparations, indicating that there was no significant M/M2 protein contamination in the HPV vector preparations (Figure 1b).

\section{HPV PsV-based induction of RSV-specific T-cell responses}

$\mathrm{BALB} / \mathrm{c}$ mice were immunized either intramuscularly (IM) or intravaginally (IVag) with HPV16 PsV encapsidating plasmid DNA expressing a gene that encodes a fusion protein of RSV M and M2 (M/M2). This was followed 4 weeks later by another immunization by the same route with the plasmid expressing M-M2 encapsidated within heterologous HPV45 $\mathrm{PsV}$ to circumvent vector-neutralizing antibody responses elicited by the priming dose (Figure 1c). Four weeks after the second immunization, mice were challenged with RSV intranasally (IN) to probe for vaccine-induced priming of $\mathrm{T}$-cell responses in the lung. Lung lymphocytes were evaluated on days 4 and 7 post-challenge (gating shown in Figure 2a). Interestingly, IM immunized as well as IVag immunized mice showed evidence of primed T-cell responses, with about 5\% of lung CD $8+\mathrm{T}$-cells specific for $\mathrm{K}^{\mathrm{d}} \mathrm{M} 2_{82-90}$ by day 4 and between 40 and $60 \%$ by day 7 (Figure $2 \mathbf{b}$ ) compared with no detectable tetramer-specific T-cell response by day 4 in empty vector-immunized mice and about $10 \%$ at day 7 (Figure $\mathbf{2 b}$ ). Therefore, both IM and IVag immunized mice were primed for RSV-specific memory CD8 + T-cell responses that were of greater magnitude and more rapid than in primary RSV infection in which the CD8 + T-cell responses typically peak between days 7 and 10 .

\section{HPV PsV induction of RSV- and HPV-specific antibody responses}

$\mathrm{M} / \mathrm{M} 2$-specific antibody was measured by kinetic ELISA in serum before the challenge and in serum, nasal wash, vaginal wash, and bronchoalveolar lavage on day 7 post-challenge (Figure 3). Mice immunized IVag had the greatest serum M/M2-specific antibody response, which was biased toward IgG2a rather than IgG1 (Figure 3a). Post-challenge, IVagimmunized mice had the greatest boost in antibody response, again strongly IgG2a-biased. Mock-immunized mice undergoing primary RSV infection did not have a significant antibody response detected by day 7 post-challenge. Typically in 
a

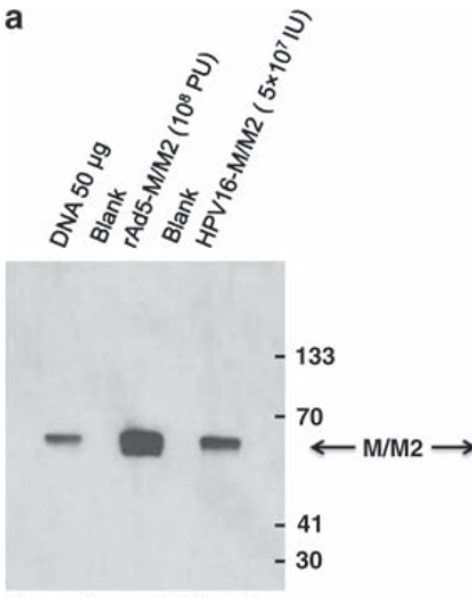

Transduced 293T cells b

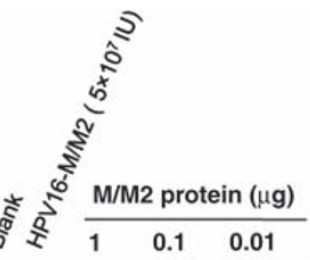

$\rightarrow$
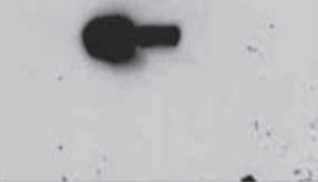

Single vaccine dose

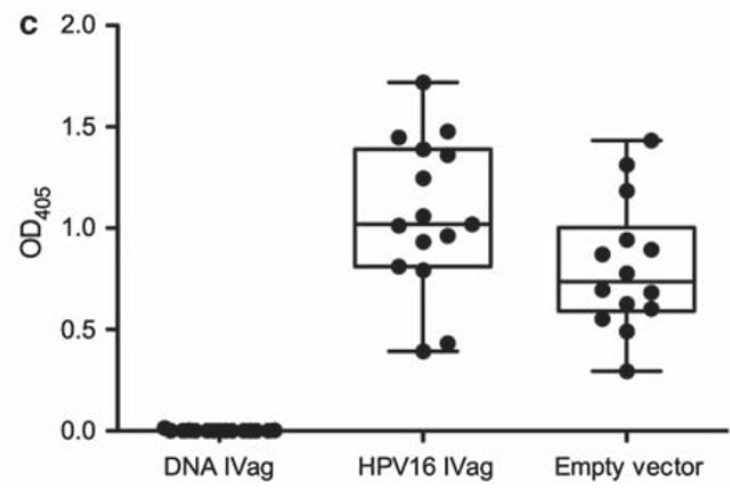

Figure $1 \mathrm{M} / \mathrm{M} 2$ expression and characterization of human papillomavirus (HPV) pseudovirion (PsV) particles. To confirm the integrity of each vector construct, M/M2 expression was tested in vitro in 293 T cells after $24 \mathrm{~h}$. p8400 VRC CMV/R plasmid was evaluated using the Invitrogen Lipofectamine 2000 (Carlsbad) protocol.

Replication-defective recombinant adenovirus serotype 5 (rAd5) and HPV-encapsidated DNA were used at an multiplicity of infection (MOI) of 5. Cell lysates in Invitrogen NuPAGE LDS sample buffer (Carlsbad) were run on $4-12 \%$ Bis Tris gels and stained with a polyclonal RSV primary antibody (Maine Biotechnology) and peroxidase-conjugated affiniPure Rabbit anti-goat IgG $(\mathrm{H}+\mathrm{L})$ secondary antibody (Jackson ImmunoResearch Labs) (a). To determine the extent of HPV particle contamination with $\mathrm{M} / \mathrm{M} 2$ protein, western blot analysis was done comparing a single dose of the HPV particles with specified amounts of purified recombinant M/M2 protein. The only difference from the western blot procedure stated above was the use of murine monoclonal antibodies to $\mathrm{M}$ and $\mathrm{M} 2$ as the primary and peroxidase-conjugated goat anti-mouse IgG $(\mathrm{H}+\mathrm{L})$ antibody (Jackson ImmunoResearch Labs) as the secondary label (b). To assess vector-specific immunity induced by the vaccination approach, HPV16-specific IgG was measured by standard ELISA (c). HPV-specific antibodies were induced by a single IVag dose of the vaccine vector, HPV PsV-M/M2, and the empty vector control, HPV PsV-luciferase.

primary infection, antibody is not easily detectable until day 14 and does not plateau until about 6 weeks post-challenge. The antibody detected in bronchoalveolar lavage fluid had the same pattern as that in serum (Figure $\mathbf{3 b}$ ), and the nasal wash had only marginally detectable levels of antibody by day 7 post-challenge in any group (Figure 3c). RSV M/M2-specific vaginal wash antibody was only detected in IVag-immunized mice (Figure 3d).

\section{Cytokine production in lungs post-challenge}

Cytokines in the supernatants from ground lungs were measured by EIA on days 4 and 7 post-challenge. Production of cytokines commonly associated with activated CD8 + T-cells responding to RSV infection, including IFN- $\gamma$, MIP- $1 \alpha$, and MIP- $1 \beta$, were much higher in immunized mice at day 4 , and by day 7 were reduced to levels less than or equal to those found in empty vector-immunized mice (Figure 4). This suggests vaccination by either the IM or the IVag route primed T-cells for a response that was both activated and regulated earlier than in mice undergoing primary infection. In addition, none of the cytokines associated with Th2 responses, including IL-4, IL-10, and IL-13 (data not shown), were detectable in lung supernatants, indicating the T-cell response in lung was composed primarily of Th1 CD4 + and CD8 + T-cells.

\section{HPV PsV-encapsidated DNA induces a different pattern of immune response than naked DNA}

Next we asked whether the HPV PsV-encapsidated plasmid could induce a significant immune response after a single immunization and whether IVag-delivered naked DNA was immunogenic. In this experiment, $\mathrm{H}-2^{\mathrm{b} / \mathrm{d}}$ hybrid CB6F1/J were used, so that both $\mathrm{K}^{\mathrm{d} M} 2_{82-90}$ and $\mathrm{D}^{\mathrm{b}} \mathrm{M}_{187-195}$ tetramers could be used to measure T-cell responses. Mice were immunized with a single IVag inoculation and challenged 4 weeks later. Blood tetramer responses for the dominant $\mathrm{M} 2_{82-90}$ responses were detected at a low level 10 days after a single IVag immunization with HPV16 PsV-M/M2 and DNA-M/M2 (data not shown). HPV16 PsV-encapsidated DNA primed for high-magnitude $\mathrm{M}_{82-90}$ and $\mathrm{M}_{187-195}$-specific T-cell responses. A single dose of $50 \mu \mathrm{g}$ naked DNA IVag also primed for a strong T-cell response in the lung 7 days post-challenge (Figure 5). However, the HPV16 PsV-immunized mice had a more balanced response between the dominant $\mathrm{M} 2_{82-90}$ and the subdominant $\mathrm{M}_{187-195}$ epitope relative to DNA-primed mice, in which the epitope response disparity was greater. The ratio of the $\mathrm{M} 2_{82-90}$ to $\mathrm{M}_{187-195}$-specific tetramer responses for mice immunized with naked DNA, HPV PsV-encapsidated DNA, or empty vector was $12 \pm 3.5,4.3 \pm 0.3$, and $4.4 \pm 0.8$, respectively ( $n=5$ per group, $P=0.06$ ). We have previously seen this effect, associated with the frequency of FoxP3 + T-cell responses, ${ }^{31}$ and indeed the frequency of FoxP3 + CD4T cells in the lungs of HPV PsV-primed mice is greater than in DNA-primed mice (data not shown). Another difference in the immune response pattern was that naked DNA-immunized mice did not have any detectable M/M2-specific antibody response in serum pre-challenge, while mice immunized with a single dose of HPV16 PsV-encapsidated DNA had detectable antibody (Figure 6a). In contrast to $B A L B / c$ mice immunized with two doses of HPV PsV vectors (Figure 3a), the relative induction of M/M2specific IgG1 and IgG2a after a single dose of HPV PSV M/M2 in the hybrid mice before the challenge was more balanced, although post-challenge the response was predominantly IgG2a. In samples derived from mice on day 12 , post-challenge M/M2-specific antibody was detected in bronchoalveolar lavage, nasal wash, and vaginal wash, only in the HPV16 PsV-immunized mice (Figure 6b-d). RSV-specific nasal wash antibody was detected at significantly higher levels in HPV16 PsV-immunized mice, indicating 

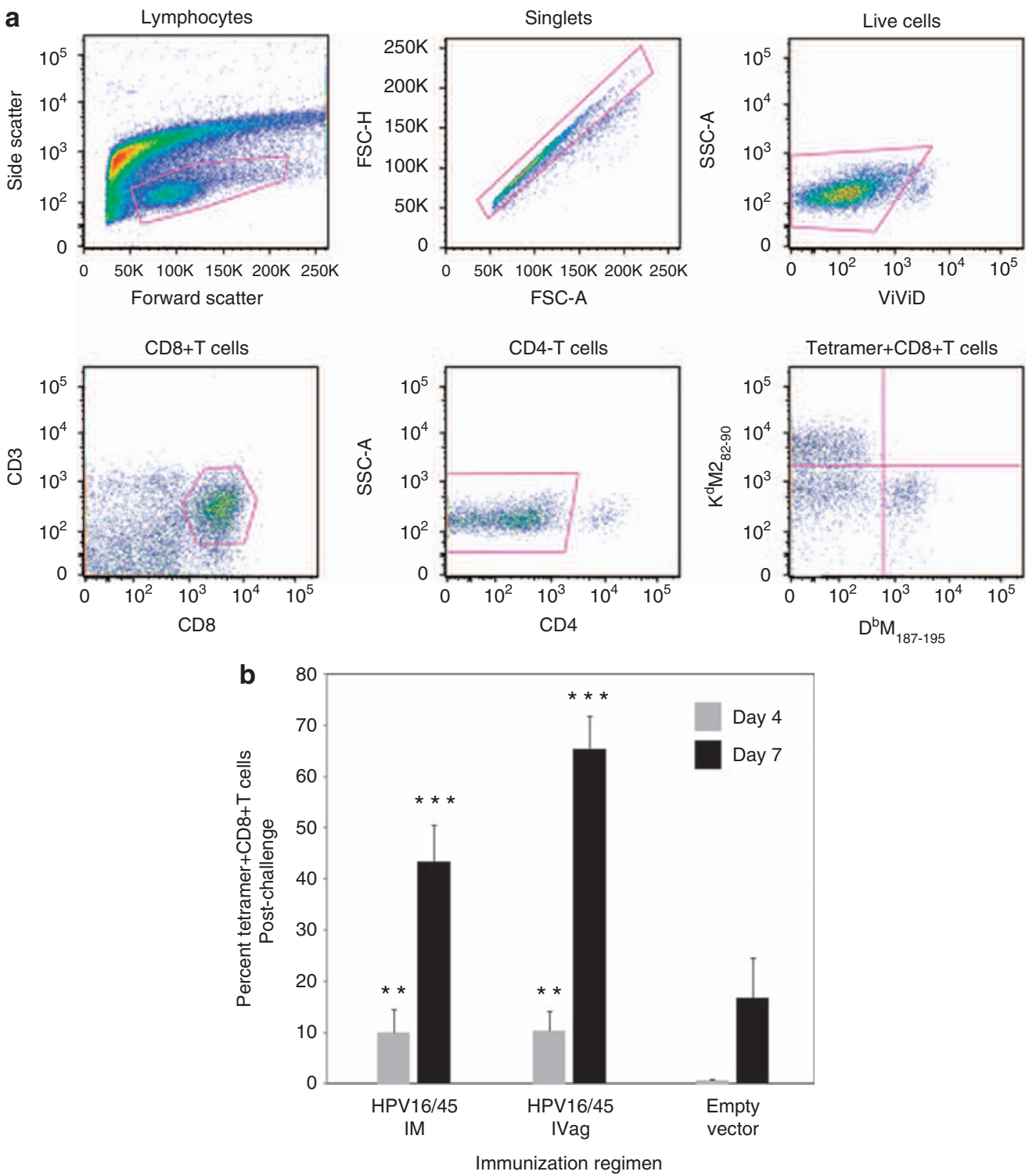

Figure 2 Tetramer responses. The gating strategy for T-cell analysis by flow cytometry is shown in panel (a). First, lymphocytes were selected by forward and side-scatter. Next, singlets were selected, and dead cells that were ViVid positive were excluded. Then, we selected CD8+CD3+ cells, and the excluded CD4 + cells before gating on tetramer-specific responses. $\mathrm{K}^{\mathrm{d}} \mathrm{M} 2_{82-90}$-tetramers were conjugated with phycoerythrin, and $D^{\mathrm{b}} \mathrm{M}_{187-195^{-}}$tetramers were conjugated with allophycocyanin, allowing them to be analyzed together in mice with both $\mathrm{H}-2^{\mathrm{d}}$ and $\mathrm{H}-2^{\mathrm{b}}$ alleles. We first evaluated BALB/c $\left(\mathrm{H}-2^{\mathrm{d}}\right)$ mice 4 weeks after immunization with $5 \times 10^{7} \mathrm{IU}$ of HPV16-M/M2 IVag or IM. Secondary immunizations of $5 \times 10^{7}$ p.f.u. HPV45-M/M2 were given at week 4 by the same route. Negative control mice received $5 \times 10^{7}$ IU HPV-luciferase intravaginally (IVag). All mice were treated with Depo-Provera (4 days) and N-9 $(5 \mathrm{~h}$ ) before IVag immunization. Respiratory syncytial virus (RSV) infection challenge occurred at week 8. (b) On days 4 and 7 following challenge with $10^{7}$ p.f.u. of A2 strain of RSV, mice were euthanized; lungs were harvested, and stained for $\mathrm{K}^{\mathrm{d}} \mathrm{M} 2_{82-90}$ tetramer-specific CD8 + T cells. Day 4 (light gray) and 7 (black) post-challenge $\mathrm{K}^{\mathrm{d}} \mathrm{M} 2_{82-90}$ tetramer-specific responses were higher in the immunized mice indicating significant priming. ${ }^{\star \star} P \leqslant 0.001 ;{ }^{* \star} P \leqslant 0.0001$ by one-way statistical analysis of variance and $t$-test compared with empty vectorimmunized control. $N$ =five mice per group.

that prior priming of the vaginal mucosa with HPV PsV elicited an RSV-specific recall response in the nasal mucosa post-challenge as well as in vaginal mucosa. Post-challenge, in both of the nasal and vaginal wash fluids, there was a greater amount of total IgG that correlated with the M/M2-specific responses in those samples (Figure 6). This suggests that IVag immunization resulted in local and systemic immunological events that increased transudation in the vagina and nose after RSV infection of the airway. This did not occur in DNA-primed mice. M/M2-specific IgA was detected in both nasal (Figure 7a) and vaginal wash (Figure 7b) post-challenge in HPV16 PsV-M/M2-immunized mice, but not in mice immunized with naked DNA. Total IgA was similar in samples from both immunized and empty vector-immunized groups, suggesting that the M/M2-specific IgA was more likely to be locally produced and not the result of transudation.

\section{HPV PsV encapsidation improves the potency of DNA immunization}

Next we asked how the relative potency of a single immunization with HPV vector compared with naked DNA immunization. 
a

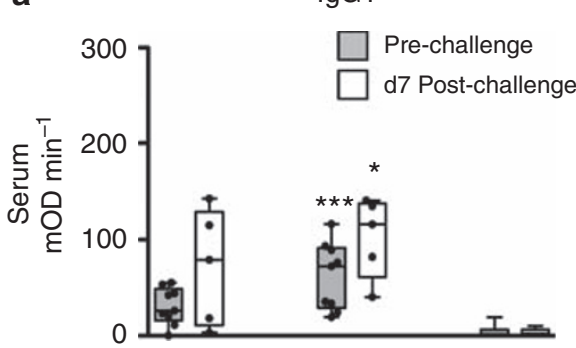

b

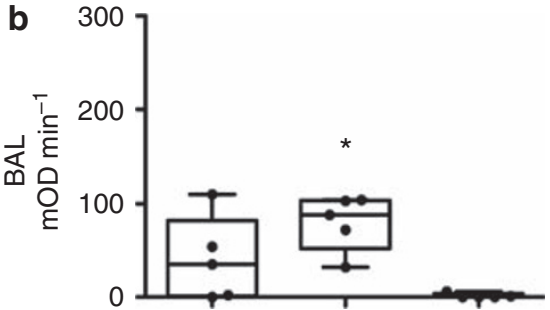

C
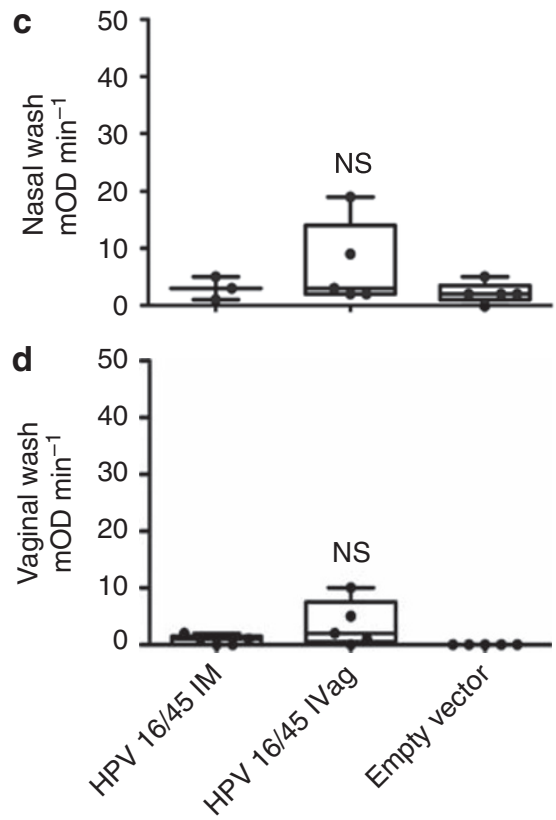

$\operatorname{lgG} 2 \mathrm{a}$
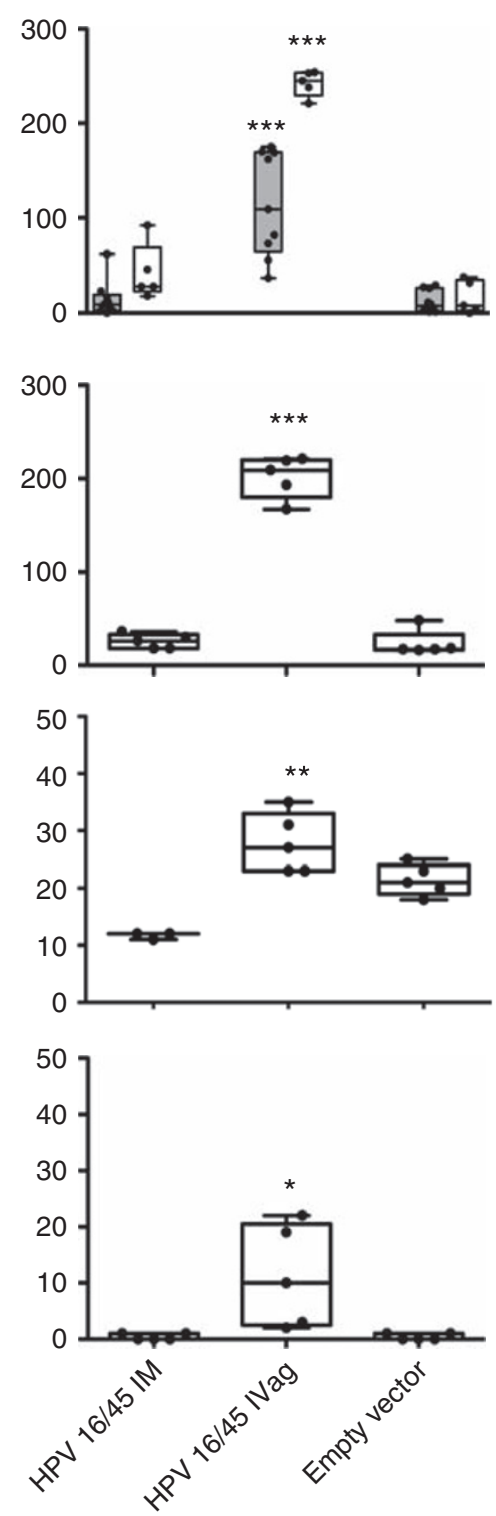

Figure 3 Antibody responses. Immunization schedule was completed as described for Figure 1, and samples were analyzed for IgG1 and IgG2a M/M2-specific antibody responses by kinetic ELISA in serum 3 days before challenge and 7 days following challenge (a). Antibody was measured in mucosal secretion 7 days after nasal challenge with respiratory syncytial virus (RSV). All immunized and empty vector-immunized mice were infected with RSV. Post-challenge IgG1 and IgG2a M/M2-specific antibody responses are shown for bronchoalveolar lavage (b), nasal wash (c), and vaginal washes (d). NS $=P>0.05 ;{ }^{*} P \leqslant 0.01 ;{ }^{\star \star} P \leqslant 0.001 ;{ }^{\star \star \star} P \leqslant 0.0001$ by one-way statistical analysis of variance and $T$-test compared with the empty vector-immunized control group. $N=5$ mice per group at each time point.

It is estimated that in a standard dose of HPV PsV particles there is about $5 \mathrm{ng}$ of plasmid DNA. Therefore, BALB/c mice were immunized once IVag with $5 \mathrm{ng}, 500 \mathrm{ng}$, or $50 \mu \mathrm{g}$ (50,000 ng) of naked DNA or $5 \times 10^{7}$ IU HPV PsV-encapsidated DNA particles. Single doses of both HPV16 and HPV45 PsV vectors were evaluated independently. Controls included an empty vector-immunized group and a group that received $1 \mu \mathrm{g}$ of M/M2 protein in combination with a non-expressing plasmid. This group was included to account for the possibility that some M/M2 protein produced during transfection might be contaminating the HPV particle preparations, although western blot analysis could not detect M/M2 present in a single dose of vaccine (Figure 1b). Mice were challenged with RSV IN 4 weeks after immunization and $\mathrm{K}^{\mathrm{d}} \mathrm{M} 2_{82-90}$ tetramer-specific CD8 + T-cell responses were measured in the lung on day 7. The HPV16 and HPV45 PsV-immunized mice were the only ones with evidence of significant RSV-specific $\mathrm{T}$-cell priming (Figure 8a). This pattern of response was also seen in measurements of serum antibody, with only HPV PsVimmunized mice having detectable levels of antibody pre-challenge (Figure 8b). By day 7 post-challenge (Figure 8c), it was clear that HPV PsV vector primed for a significantly greater antibody response. These data suggest that HPV PsV encapsidation of plasmids improved the efficiency of delivery, expression, and immunogenicity of the vaccine antigen in target cells. 


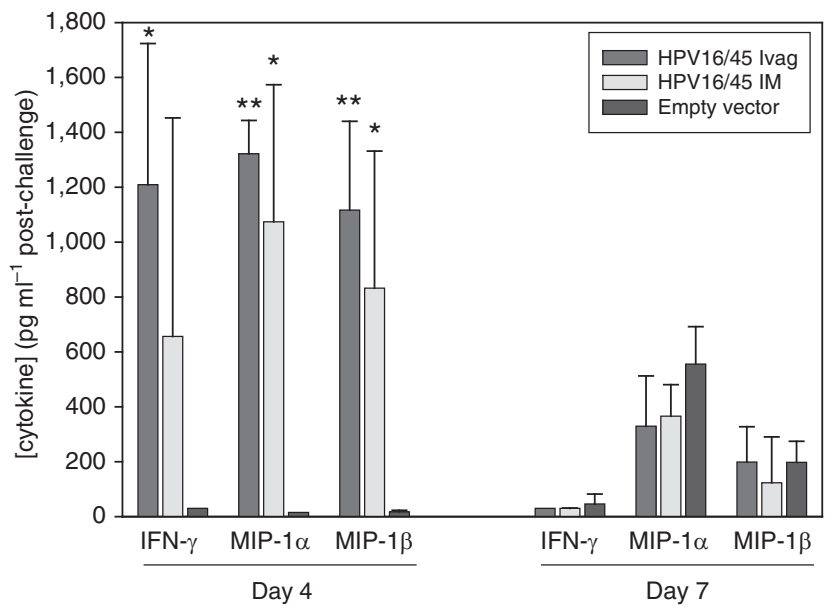

Figure 4 Cytokine detection. Immunization schedule was completed as described for Figure 1. On days 4 and 7 following challenge, IFN- $\gamma$, MIP- $1 \alpha$, and MIP- $1 \beta$ cytokines were detected in lung supernatants by EIA. IL-4, IL-10, and IL-13 were below the limit of detection $\left(20 \mathrm{pg} \mathrm{ml}^{-1}\right)$. ${ }^{\star} P \leqslant 0.01$ and ${ }^{* \star} P \leqslant 0.001$ by $t$-test compared with the empty vectorimmunized control group. $N=5$ mice per group at each time point from one independent experiment.

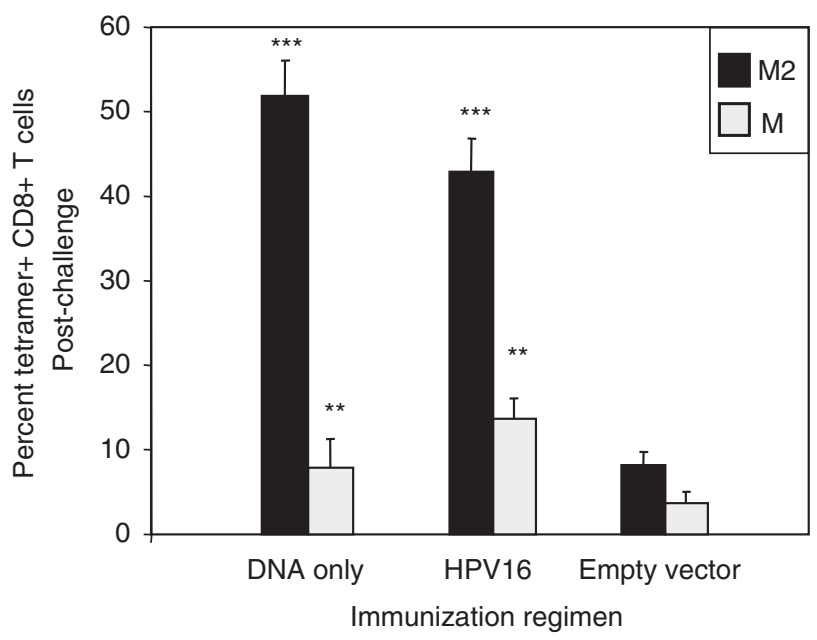

Figure 5 Potency of T-cell induction by human papillomavirus (HPV) pseudovirion(PsV)-encapsidated DNA. CB6F1/J mice were immunized IVag with $5 \times 10^{7}$ IU HPV16-PsV-M/M2 or $50 \mu$ g naked M/M2 DNA. Negative control mice received $5 \times 10^{7}$ IU HPV16 PsV-luciferase. All mice were challenged IN with respiratory syncytial virus (RSV) at week 4. $D^{\mathrm{b}} \mathrm{M}_{187-195}$ (grey) and $\mathrm{K}^{\mathrm{d}} \mathrm{M} 2_{82-90}$-specific (black) CD8 + T-cells were detected by tetramer staining in lungs on day 7 post-challenge. ${ }^{\star} P \leqslant 0.01$ and ${ }^{* *} P \leqslant 0.001$ by one-way statistical analysis of variance and $t$-test compared with empty vector-immunized mice. $N=5$ mice per group from one independent experiment.

\section{Intravaginal HPV delivery of plasmids results in high levels of self-limited antigen expression}

To better understand how the magnitude and duration of antigen expression were affected by the delivery approach, plasmids were constructed to express firefly luciferase, and in vivo light emission was measured after IM and IVag delivery of HPV PsV or naked DNA. As signal is lost within a few hours of substrate administration, we were able to image the same mice for each of the daily time points. Luciferase expression from IVag-administered
HPV PsV was significantly higher than that observed with naked DNA delivery on days 1 and $2(P<0.001$ by statistical analysis of variance) (Figure 9). Expression from HPV PsV IVag delivery peaked within 2 days and returned to background levels by day 7. Expression from naked DNA was much lower, despite delivery of 10,000-fold more copies of the same plasmid, peaking on day 1 and returning to near-background levels by day 3. Although expression from IVag naked DNA was relatively low, it was significantly above background ( $P<0.05$ by Dunn's method). The initial strong burst of antigen after IVag instillation of HPV PsV could account for the differences observed in the immunological responses between HPV PsV and naked DNA.

Mice inoculated IM with naked DNA exhibited a higher level of luciferase expression than those inoculated with HPV PsV luciferase-expressing plasmids during the first 7 days after injection $(P<0.001$ by statistical analysis of variance). Luciferase expression after IM delivery of naked DNA peaked during the first week and then plateaued at about $50 \%$ of peak. In contrast, IM-delivered HPV PsV resulted in delayed expression that did not reach a similar level of expression compared with naked DNA until about day 7. Surprisingly, luciferase expression continued with slow increase over subsequent weeks in animals receiving HPV PsV-encapsidated DNA IM.

To better understand the basis for improved immune responses from IVag delivery of HPV PsV-encapsidated DNA, naked DNA plasmids expressing red fluorescent protein (RFP) or the same plasmid encapsidated in HPV PsV were intravaginally instilled into mice. On day 2, genital tracts were removed, frozen, and sectioned for evaluation of RFP signal as readout for gene transduction by fluorescent microscopy. RFP-positive epithelial cells were easily identified in the HPV PsV-encapsidated DNA recipients (Figure 9b). Very rare cells could be identified in the naked DNA recipients. However, the morphology of RFP-positive cells suggests that they are also epithelial cells (Figure 9c). Nevertheless, it is possible that other cells such as mobile antigen-presenting cells had already left the tissue. These data are consistent with the light emission data, and suggest that the higher magnitude of gene delivery to and antigen expression in keratinocytes is a major factor in the immunogenicity of HPV PsV delivery of DNA.

\section{DISCUSSION}

Mucosal immunization may be important for protection against viral pathogens such as HIV, herpes simplex virus, or RSV. While parenteral immunization can elicit protective immune responses in animal models of these virus infections, effective immunization of humans has been difficult. Each of these pathogens has multiple features that make them difficult vaccine targets, ${ }^{32}$ but one feature they share in common is that transmission usually occurs across a mucosal surface. Mucosal immunization against viruses has traditionally been accomplished by using live-attenuated viruses. However, for viruses like HIV or herpes simplex virus that can cause latent or persistent infection, or RSV that infects neonatal airways, the use of replication-competent virus presents a number of safety concerns. Gene-based vector delivery of vaccine antigens affords an option for eliciting immune responses against authentic antigenic structures, while avoiding some of the liabilities of 

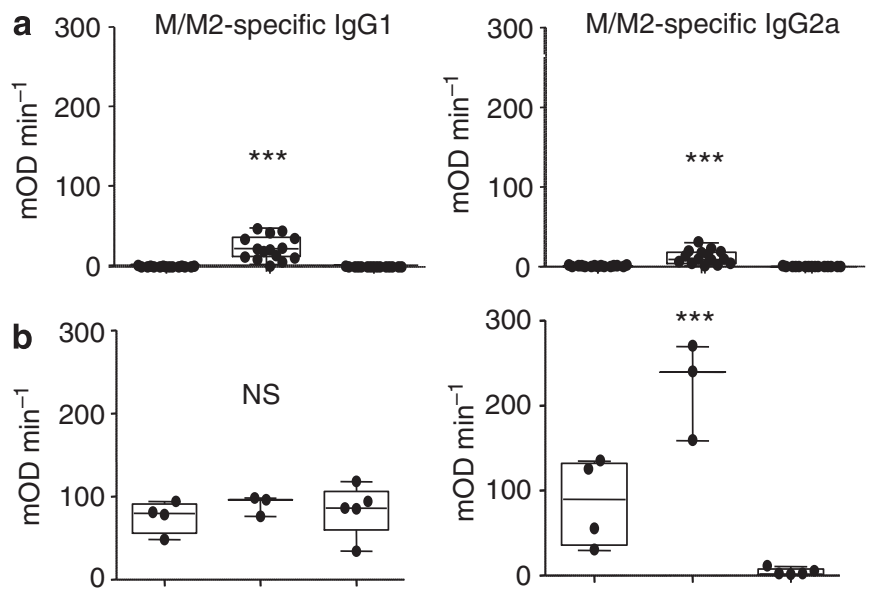

Total IgG
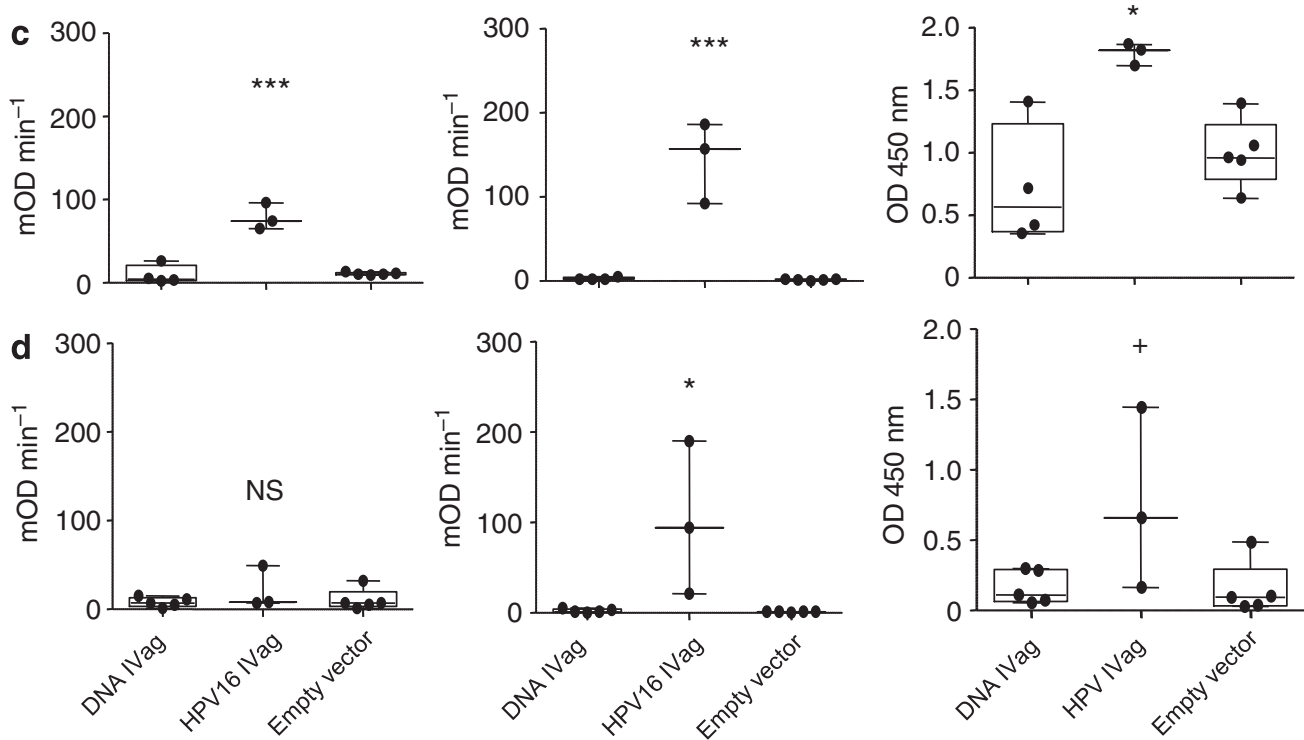

Figure 6 Induction of IgG by human papillomavirus (HPV)-encapsidated DNA. Following immunization with a single dose of HPV16 PsVencapsidated M/M2-expressing DNA, naked M-M2-expressing DNA or empty HPV16 PsV capsids M/M2-specific IgG1 and IgG2 levels were evaluated by kinetic ELISA in serum 3 days before challenge (a). Respiratory syncytial virus (RSV) M/M2-specific IgG isotypes were also measured in bronchoalveolar lavage (b), nasal washes (c), and vaginal washes (d) on day 12 post-challenge. Total IgG was measured by ELISA in both nasal and vaginal washes (c and d, panels on the far right). $P$ values are derived from one-way statistical analysis of variance $\left(\mathrm{NS}=P>0.05 ;{ }^{+} P \leqslant 0.05\right.$; $\left.{ }^{\star} P \leqslant 0.01 ;{ }^{\star \star} P \leqslant 0.001 ;{ }^{\star \star \star} P \leqslant 0.0001\right)$. The data represent each individual sample from each animal from one independent experiment.

the native viral pathogen. However, there have been relatively few options for direct mucosal immunization with the genebased vaccine vectors described, and they have primarily been replication-competent vectors based on adenovirus, ${ }^{33}$ picornavirus, ${ }^{34}$ rhinovirus, ${ }^{35}$ or paramyxovirus. ${ }^{36,37}$ Replication-defective poxvirus and adenovirus vectors have been delivered mucosally to humans, but with limited success. ${ }^{5,6}$ Here, we describe the mucosal delivery of DNA facilitated by HPV PsV encapsidation. IVag delivery of HPV PsV-encapsidated DNA induces both T-cell and antibody responses to the antigen encoded by the plasmid. HPV PsV encapsidation appears to increase the relative potency of the DNA vaccine, based on gene copies delivered, and induces stronger antibody responses than DNA alone.

IVag delivery was more immunogenic than IM. Typically, replication-defective gene-based vectors have been less potent when delivered mucosally than when delivered parenterally. The mucosal advantage observed in this report may be because of the natural tropism of HPVs for basal epithelium, ${ }^{7}$ but may also reflect access to other antigen-presenting cells in the mucosa that are not present in the muscle. Alternatively, there may be selected cells in the mucosa that can recognize the pathogen-associated molecular patterns in HPV resulting in toll-like receptor activation and an adjuvantlike effect that does not happen in the muscle environment. The higher ratio of IgG1 to IgG2a and the reduced ratio of $\mathrm{K}^{\mathrm{d}} \mathrm{M} 2_{82-90}$ to $\mathrm{D}^{\mathrm{b}} \mathrm{M}_{187-195}$ tetramer responses in the IM immunized group compared with the IVag immunized group suggests that a different pattern of CD4 T-cell response was elicited by the two routes. Higher IgG1 is associated with IL-4 production from Th2 responses, and a lower disparity in the epitope hierarchy is associated with Treg responses, ${ }^{31}$ and both suggest that differences in antigen presentation occurred between IM and IVag-administered particles.

Delivery of naked DNA to the disrupted epithelium was also immunogenic, although the copy number of the gene encoding the M-M2 antigen was 10,000-fold higher in the naked DNA 

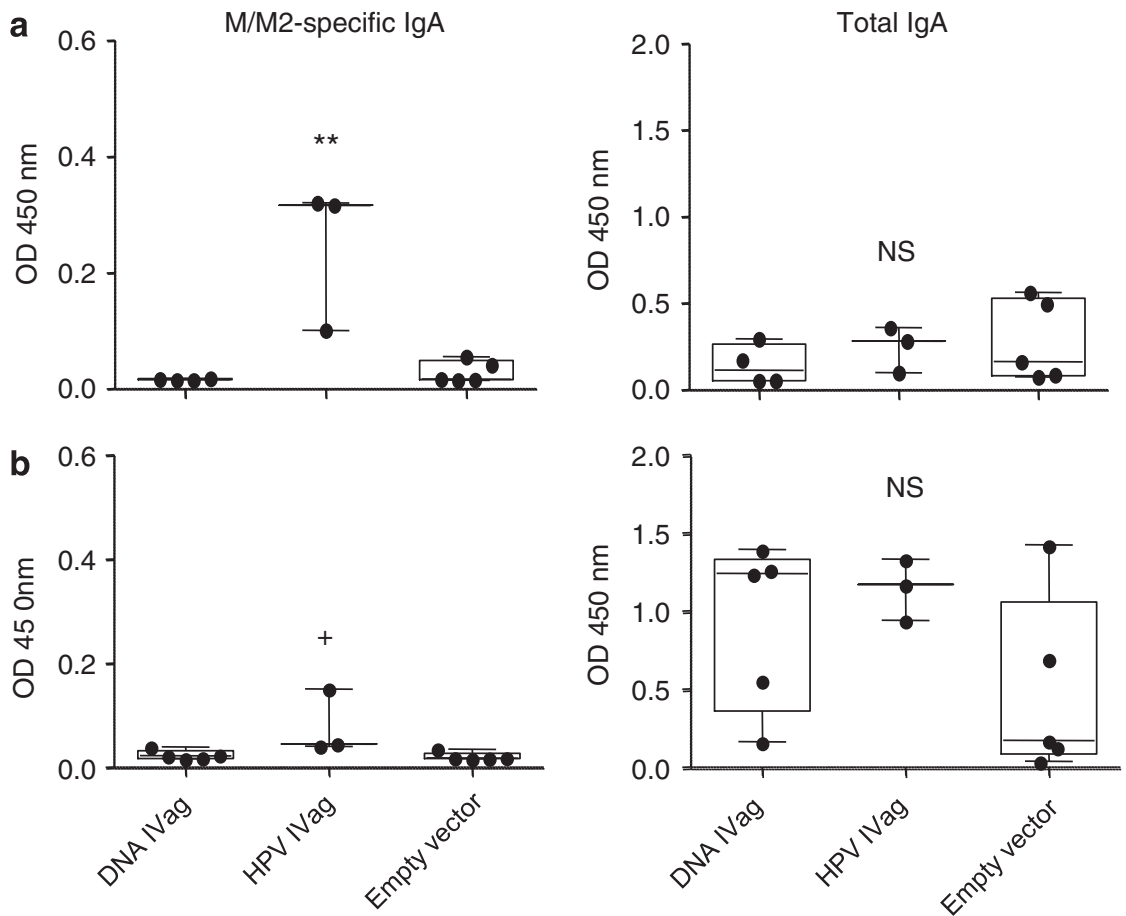

Figure 7 Induction of IgA by human papillomavirus (HPV)-encapsidated DNA. Mice immunized with a single dose of HPV16 PsV-encapsidated M-M2-expressing DNA, naked M/M2-expressing DNA, or empty HPV16 PsV capsids were challenged with respiratory syncytial virus intranasally. Nasal washes (a) and vaginal washes (b) were evaluated for M/M2-specific IgA or total IgA by ELISA on day 12 post-challenge. $P$ values are derived from one-way statistical analysis of variance (NS $\left.=P>0.05 ;{ }^{+} P \leqslant 0.05 ;{ }^{\star} P \leqslant 0.01 ;{ }^{\star \star} P \leqslant 0.001\right)$. $N=5$ mice per group from a single independent experiment.

dose than in a dose of HPV-encapsidated plasmids. This and recent in vivo studies showing improved potency of DNA immunization by electroporation ${ }^{38}$ suggest that the major limitation for DNA vaccination is at the level of delivery into cells and not at one of the many downstream points ultimately required for expression of the vaccine antigen.

IVag delivery of naked DNA was unable to elicit M-M2specific antibody responses. In contrast, HPV-encapsidated DNA-delivered IVag-induced antibody responses in the vaginal epithelium and primed for antibody responses in the upper airway. This could involve delivery to alternative target cells and consequently different antigen-processing and presentation pathways, a different pattern of toll-like receptor stimulation, or a different threshold of antigen expression needed for T-cell vs. antibody induction.

We asked whether the magnitude and kinetics of vaccine antigen availability could explain the distinct properties in the immune response elicited by DNA-delivered IVag or IM, either encapsidated with HPV PsV or not. Using luciferase expression as a surrogate for vaccine-antigen expression, ${ }^{39}$ we found that IVag delivery of DNA by HPV PsV resulted in significantly higher antigen production, but for a brief circumscribed period of time. This transient expression pattern may be attributed to the tropism of HPV-targeting basal epithelial cells, which would be expected to differentiate and be sloughed off into the vaginal lumen over the course of about 5 days. Based on the light emission and RFP expression data, we favor the explanation that IVag HPV PsV improves gene delivery and the magnitude of antigen expression and that antibody is more dose-dependent than T-cell responses.
IM delivery of the HPV PsV-encapsidated DNA had weak expression in the early days after inoculation. Delivery of naked DNA resulted in better expression when delivered IM than mucosally, and luciferase expression was prolonged. However, the level of luciferase expression was much lower than with HPV PsV-encapsidated DNA, and the delayed clearance that suggested different target cells were transduced. IVag expressed antigen appears to be immediately available in basal epithelial cells, and based on its rapid clearance, is probably cross-presented by dendritic cells in the mucosa. IM delivered HPV-encapsidated plasmid results in slower, more cumulative production of antigen, but the target cell for infection and method of antigen presentation are unclear.

The demonstration of IgA in both nasal and vaginal wash indicates that HPV PsV delivery induced a mucosal antibody response to the antigen encoded by the encapsidated plasmid, and that the vaginal mucosa serves as a local inductive site for the response. In addition to direct induction of IgA in vaginal mucosa, there was evidence that IgA responses in nasal secretions were primed by IVag immunization, suggesting mobility of the adaptive mucosal response. It was also notable that total $\operatorname{Ig} G$ was increased in nasal and vaginal washes post-challenge. The evidence of increased transudation at the site of infection and at the site of original immunization, suggests that immune effectors were activated at both sites during infection of the airway, and that HPV PsV vector immunization of the vaginal mucosa can induce locally persistent adaptive responses.

HPV PsV-delivered IVag primes for a similar pattern of cytokine response in the lung after RSV challenge as other 

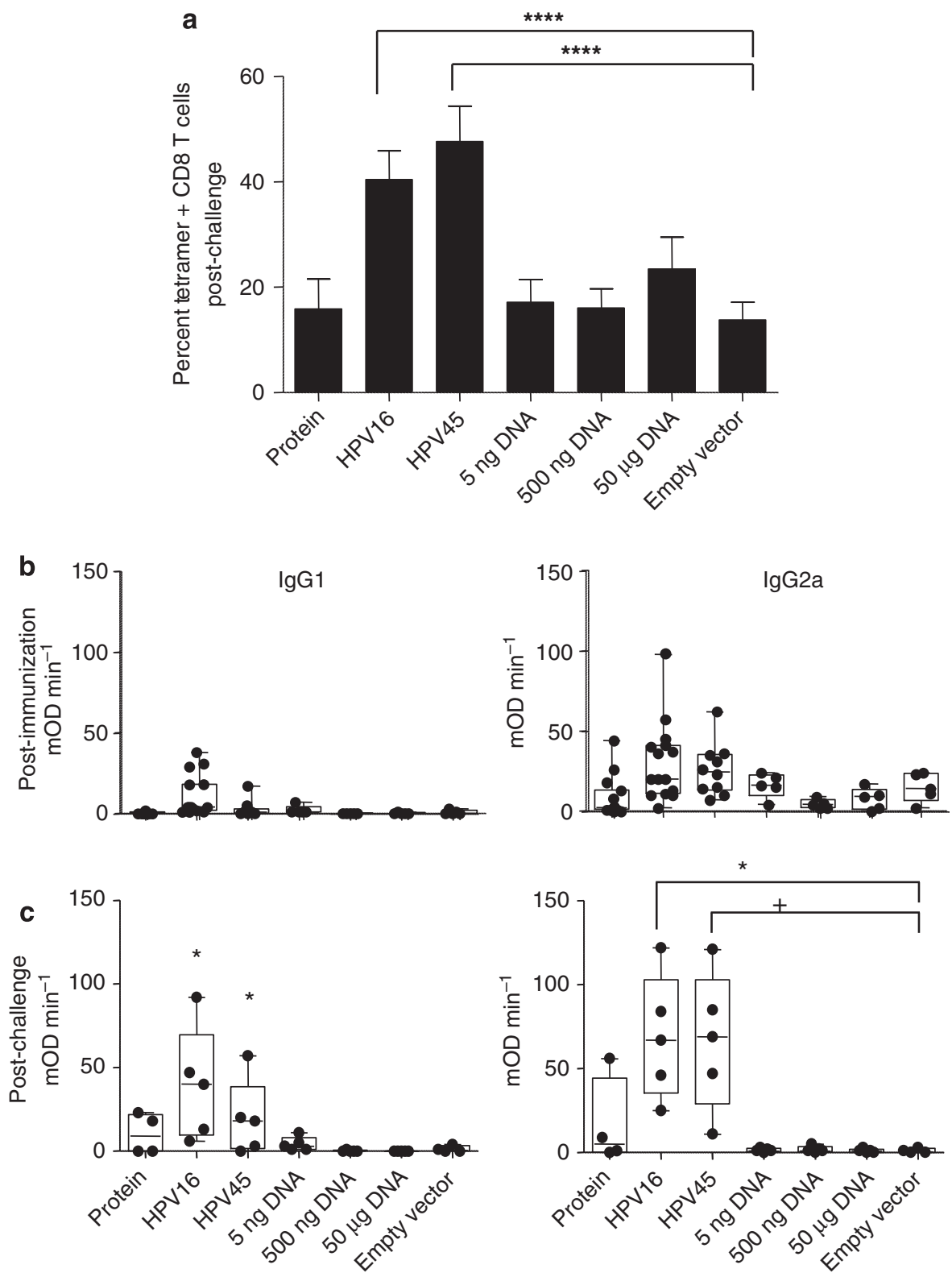

Figure 8 Human papillomavirus (HPV) pseudovirion (PsV)-encapsidation improves the delivery of plasmid DNA. BALB/c mice were immunized once intravaginally (IVag) with a single $5 \times 10^{7} \mathrm{IU}$ dose of HPV16 PsV-M/M2, 5×107 IU HPV45 PsV-M/M2, $5 \mathrm{ng}, 500 \mathrm{ng}$, or $50 \mu \mathrm{g}$ of naked M/M2 DNA, or $1 \mu \mathrm{g}$ of M/M2 purified protein. Negative control mice received $5 \times 10^{7} \mathrm{IU}$ HPV-luciferase IVag. All mice were challenged IN with RSV at week 4 .

Seven days after challenge, M and M2-specific CD8 + T cells in lung were measured by tetramer staining (a) and M/M2-specific IgG1 and IgG2 serum responses were measured by kinetic ELISA pre-challenge (b) and post-challenge (c). $P$ values represent one-way statistical analysis of variance and selected comparisons by $t$-test $\left({ }^{+} P \leqslant 0.05 ;{ }^{\star} P \leqslant 0.01 ;{ }^{* * \star \star} P \leqslant 0.00001\right) . N=5$ mice per group for each post-challenge timepoint from one independent experiment.

parenterally administered gene-based vectors with no evidence of Th2 cytokines. For RSV in particular and for viral vaccines in general, it is desirable to avoid the induction of Th2 responses. The cells and effector molecules associated with Th2 responses can lead to diminished CD8T-cell function, altered antibody isotypes, and pathology resembling allergic inflammation. ${ }^{40,41}$ This pattern of response has been associated with reduced efficacy and vaccine-induced immunopathology. ${ }^{42}$

The major limitation of this approach is that disruption of the vaginal epithelium is required for HPV to access its target cells. Nonoxynol-9 is a licensed, over-the-counter spermicide that was used to disrupt the epithelium. It may be clinically acceptable for limited use before a vaccination, although repeated use would be unacceptable because of the increased susceptibility to HIV-1 and perhaps other sexually transmitted diseases. ${ }^{43}$ In this test-of-concept study, mice were also pretreated with Depo-Provera to thin the vaginal epithelium before nonoxynol-9 treatment, and this would not be a clinically acceptable component of a vaccination regimen. Therefore, advancing this concept will require the development of a clinically acceptable approach for providing HPV transient access to its target cells in the basal epithelium. 


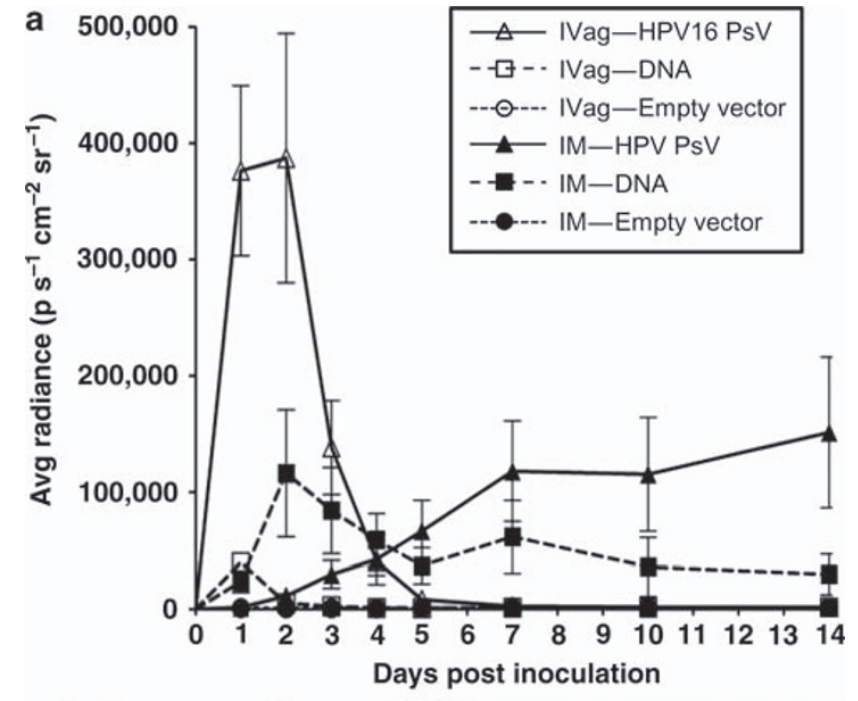

b
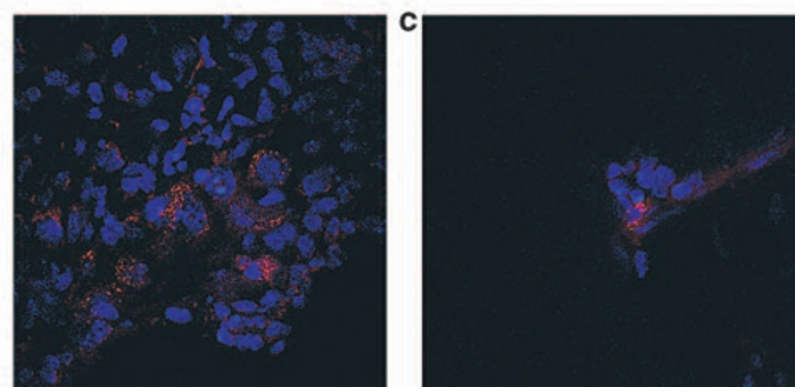

Figure 9 Temporal expression of Human papillomavirus (HPV)pseudovirion (PsV)-delivered antigen. Mice were inoculated intravaginally (IVag) $(\square, \triangle)$ or IM $(\boldsymbol{\square}, \mathbf{\Delta})$ with either pClucF (firefly luciferase) plasmid DNA $(\boldsymbol{\square}, \square)$ or HPV16 pseudovirions-encapsidated $\operatorname{pClucF}(\triangle, \boldsymbol{\Delta})$. Negative control mice received a 1:1 combination of naked and PsVencapsidated plasmid expressing red fluorescent protein (RFP IVag, $\bigcirc$; $\mathrm{IM}, \bullet)$. Luciferin substrate $\left(15 \mathrm{mg} \mathrm{ml}^{-1}\right)$ was administered IVag $(20 \mu \mathrm{l})$ or IM $(50 \mu \mathrm{l})$ and after $3 \mathrm{~min}$ mice were imaged for bioluminescence in an IVIS 100 for 60 s with medium binning. The data were quantified as the average radiance within a standardized region of interest using Living Image software. Data represent five mice per group and are representative of two independent experiments (a). In parallel experiments, mice were inoculated with an RFP-expressing plasmid or HPV PsV encapsidating the same plasmids. After 2 days genital tracts were removed and sectioned transversely to define the location of RFP expression. Delivery by HPV16 PsV encapsidation resulted in a higher frequency of transduction with RFP-expression detected in every tissue section examined (between 50-150 infectious events per section) (b), while cells transduced by delivery of naked DNA plasmid were rare; only two infected cells were detected in all sections from the four mice examined (c).

We have described a vaccine delivery approach using HPV PsV-encapsidated plasmid DNA that results in significant levels of mucosal-antigen expression for a brief period of time and is likely to induce immune responses primarily through cross-presentation of transduced vaginal epithelium. It has the capacity to not only induce immunity against antigens expressed from the plasmid, but also provide immunity against the HPV serotype used for the delivery. This is a novel vaccine approach that merits additional investigation, especially for pathogens that infect across mucosal surfaces, and particularly for sexually transmitted diseases in women.

\section{METHODS}

Design and construction of the M/M2 fusion gene. With dominant CTL epitopes of RSV M and M2 proteins identified in BALB/C and C57BL/6 mice, respectively, ${ }^{16,17}$ a model antigen incorporating both of these target proteins was designed. A codon-modified (GeneOptimizer by GeneArt, Regensburg, Germany) gene encoding the M protein (NCBI sequence number AAB86677) was connected to a codonmodified gene encoding the M2 protein (NCBI sequence number AAB86660) by glutamine-alanine linker. The resulting fusion gene is termed "M/M2" hereafter.

Construction of DNA plasmid vectors. The RSV M/M2 gene was cloned into plasmid DNA designed for expression in mammalian cells (pVRC8400) using 5' SalI and 3' BamHI restriction sites as described. ${ }^{44}$ Plasmid inserts were sequenced after cloning to verify sequence conservation.

Production of HPV PsVs. In all 293TT cells were grown in Dulbecco's modified Eagle's medium (Gibco, Carlsbad, CA) supplemented with 10 $\%$ fetal bovine serum (Sigma-Aldrich, St Louis, MO). The PsV production and Optiprep purification method was previously reported, and is also available at http://ccr.cancer.gov/staff/links.asp?profileid=5637. Maps of HPV16 and HPV45 PsV packaging plasmids (p16sheLL and $\mathrm{p} 45 \mathrm{sheLL}$, respectively) and expression vectors for luciferase (pCLucf), $\mathrm{M} / \mathrm{M} 2$ (pCMMf), and RFP (pCIR), all driven by the CMV promoter, can also be found at this website. Purified PsV was titrated on 293TT cells by flow cytometric assessment of the expression of green fluorescent protein, as described in Buck and Thompson and titer is calculated as IU/ml.

Western blotting for expression testing. Each lot of M-M2 fusion proteins in pVRC8400 and from HPV16 and HPV45 pseudovirus was tested by western blotting before evaluation in mice. For expression analysis, 293T cell lysates in Invitrogen NuPAGE LDS sample buffer (Carlsbad, CA) were run on $4-12 \%$ Bis Tris gels and probed with goat polyclonal (Maine Biotechnology, Portland, ME) or murine monoclonal primary antibodies and peroxidase-conjugated rabbit anti-goat or goat anti-mouse $\operatorname{IgG}(\mathrm{H}+\mathrm{L})$ secondary antibodies (Jackson ImmunoResearch Labs, West Grove, PA).

Mice and experimental procedures. Female 6-8-week-old BALB/c and CB6F1 mice (Jackson Laboratories, Bar Harbor, ME) were housed in the NIAID animal care facility under pathogen-free conditions and maintained on standard rodent chow and water supplied ad libitum, according to the current ACUC approved documents.

Four days before IVag immunization mice were treated with $3 \mathrm{mg}$ DepoProvera in $100 \mu \mathrm{l}$ PBS to thin the epithelium. Five hours before immunization the vaginal epithelium was disrupted by treatment with $50 \mu \mathrm{l}$ of nonoxynol-9 $\left(40 \%\right.$ in distilled $\mathrm{H}_{2} 0$ ) diluted to a final concentration of $4 \%$ in $4 \%$ carboxymethylcellulose (CMC) (Sigma-Aldrich \#C4888) for viscosity, as previously described. ${ }^{29}$ Prior to Depo-Provera treatment, the estrous cycle of the mice was synchronized using the Whitten effect. ${ }^{46}$ Mice were immunized IVag with $5 \times 10^{7} \mathrm{IU}$ of HPV PsV-M/M2, HPV PsV-luciferase (empty vector) or naked DNA-M/M2 diluted to a final volume of $20 \mu \mathrm{l}$ in $4 \%$ carboxymethylcellulose and introduced with a positive displacement pipette. IM inoculations were given in the quadriceps. Four weeks after primary immunization, mice were boosted with a vector comprised of an alternative HPV serotype or challenged intranasally with RSV. Mice that received secondary immunizations were challenged 4 weeks after boost. Anesthetized mice were challenged with $10^{7}$ p.f.u. of A2 strain of RSV IN and evaluated as previously reported.$^{47}$ Bronchoalveolar lavage was performed as previously described in ref. 48, except that 1\% BSA in PBS was used. Following the bronchoalveolar lavage procedure, nasal wash was performed by inserting the endotracheal tube through the incision in the trachea into the nasopharynx. The nasopharynx was then flushed with $0.2 \mathrm{ml}$ PBS $+1 \%$ BSA, collecting the wash fluid from the nostrils. Vaginal washes were performed by rinsing the vaginal vault twice with $50 \mu \mathrm{l}$ of PBS. 
Blood tetramer staining. In all, $250 \mu$ of whole blood was lysed in $1 \mathrm{ml}$ ACK lysing buffer (Quality Biologicals, Gaithersburg, MD) for $7 \mathrm{~min}$. Samples were washed with $2 \mathrm{ml}$ PBS and centrifuged at 1500 r.p.m for $5 \mathrm{~min}$. Lysing and washing was repeated up to three times until the cell pellet was devoid of visible amounts of RBCs. After final removal of the supernatant, samples were stained according to the protocol for the surface and tetramer staining described below.

Surface and tetramer staining of lymphocytes. For all experiments, lungs were harvested from euthanized mice on days 4 and 7 postinfection. Lymphocytes were isolated with Fico-Lite (Atlanta Biologicals, Atlanta, GA), and tetramer-stained with $\mathrm{K}^{\mathrm{d}} \mathrm{M} 2_{82-90}$ tetramer or $\mathrm{D}^{\mathrm{b}} \mathrm{M}_{187-195}$ tetramer complexes and antibodies to $\mathrm{CD} 3, \mathrm{CD} 4$ and CD8 as previously described and analyzed by nine-color flow cytometry as previously described, ${ }^{49}$ using ViViD (Invitrogen) staining to exclude the dead cells. Flow Jo version 8.7.3 was used to analyze the data.

Measurement of M/M2-specific antibody response by kinetic ELISA. RSV M-M2 fusion protein was produced in Escherichia. coli by the Protein Expression Laboratory (SAIC, Frederick, MD). M-M2 protein was diluted in carbonate buffer ( $\mathrm{pH} 9.6$ ) and coated overnight at $4^{\circ} \mathrm{C}$ on 96-well flat-bottom ELISA plates (Nunc, Rochester, NY) at a concentration of $80 \mathrm{ng}$ well $^{-1}$. Plates were washed four times with wash buffer $(0.02 \%$ Tween-20 in PBS) using an automated plate washer (BioTek Instruments, Winooski, VT), and incubated with blocking buffer (2\% BSA in PBS) for $1 \mathrm{~h}$ at $37^{\circ} \mathrm{C}$. In all, $100 \mu \mathrm{l}$ of diluted test sample and positive control were added to each well in triplicate (two coated wells and one uncoated well). Plates were incubated for $1 \mathrm{~h}$ at $37^{\circ} \mathrm{C}$, washed and incubated for $1 \mathrm{~h}$ at $37^{\circ} \mathrm{C}$ with HRP-conjugated goat anti-mouse IgG1 (1:18,000), HRP-conjugated goat anti-IgG2a $(1: 8,000)$ and HRP-conjugated rabbit anti-mouse IgG + IgM $(1: 20,000)$ (Jackson ImmunoResearch Laboratories), or HRP-conjugated goat anti-mouse IgA $(1: 8,000)$ (Southern Biotech, Birmingham, AL). Plates were washed with wash buffer four times followed by distilled water. A volume of $100 \mu \mathrm{l}$ of Super AquaBlue ELISA substrate (eBioscience, San Diego, CA) was added to each well and plates were read immediately using a Dynex Technologies microplate reader (Chantilly, VA). The rate of color change in $\mathrm{mOD} \mathrm{min}^{-1}$ was read at a wavelength of $405 \mathrm{~nm}$ every $9 \mathrm{~s}$ for $5 \mathrm{~min}$, with the plates shaken before each measurement. The mean $\mathrm{mOD} \mathrm{min}^{-1}$ reading of duplicate wells was calculated, and the background $\mathrm{mOD} \mathrm{min}^{-1}$ was subtracted from the corresponding well.

Measurement of M-M2-specific IgA and total $\lg G$ and $\lg A$ in mucosal secretions. M-M2 was coated as described above. For total immunoglobulin (IgG + IgA), anti-kappa + anti-lambda purified antibodies (Southern Biotech) were coated at $0.1 \mu \mathrm{g} \mathrm{well}^{-1}$ each. For total IgA, $0.1 \mu$ g well $^{-1}$ of anti-IgA (Southern Biotech) was used. Plates were washed (PBS/Tween $0.05 \%$ ) once with and blocked for $2 \mathrm{~h}$ at room temperature with $0.5 \%$ milk and $0.1 \%$ fetal bovine serum in PBS. After washing twice, samples were diluted 1:10 for M-M2-specific antibody and 1:50 for total immunoglobulin content, in $0.5 \%$ milk in PBS and plates were incubated for $2 \mathrm{~h}$ at room temperature. After washing three times, secondary antibodies (goat anti-IgG or anti-IgA HRP-conjugated antibodies) (Southern Biotech) diluted in 0.5\% milk in PBS were added for $1 \mathrm{~h}$. After washing 3 times, the TMB Microwell Peroxidase Substrate System (KPL, Gaithersburg, MD) was added for $7 \mathrm{~min}$ at room temperature, treated with an equal volume of $1 \mathrm{~N} \mathrm{HCl}$, and absorbance was read at $450 \mathrm{~nm}$.

Measurement of HPV-specific antibody response. HPV16 L1 particles produced in SF9 cells ${ }^{50}$ were diluted to a concentration of $4 \mu \mathrm{g} \mathrm{ml}^{-1}$ in $1 \times$ PBS and $50 \mu \mathrm{l}$ was added to each well of a 96-well Immulon $2 \mathrm{HB}$ flat-bottom microtiter plate (Thermo, Pittsburgh, PA). Plates were incubated at $37^{\circ} \mathrm{C}$ for $2 \mathrm{~h}$, then washed three times with $1 \times \mathrm{PBS}$ followed by the addition of $50 \mu$ l blocking buffer $(0.5 \%$ dry milk, $0.1 \%$ fetal bovine serum in $1 \times \mathrm{PBS})$ and incubated at $4^{\circ} \mathrm{C}$ overnight. Plates were washed three times with $1 \times$ PBS. Experimental samples and controls were diluted 1:100 in dilution buffer $(0.5 \%$ dry milk in $1 \times$ PBS $) .50 \mu$ of diluted samples were added to the plate in duplicate and allowed to incubate for $2.5 \mathrm{~h}$ at room temperature on a nutator rocker. Plates were washed five times with $1 \times$ PBS and incubated for $1 \mathrm{~h}$ by gently rocking at room temperature with $50 \mu \mathrm{l}$ peroxidase conjugated donkey anti-mouse IgG in dilution buffer $(1: 5,000)$ (Jackson ImmunoResearch Laboratories). Plates were washed three times with $1 \times$ PBS and developed in the dark for $45 \mathrm{~min}$ at room temperature using ABTS substrate $\left(1 \mathrm{mg} \mathrm{ml}^{-1}\right.$ ) (Roche, Indianapolis, IN). Absorbance was read at $405 \mathrm{~nm}$ with a reference set at $492 \mathrm{~nm}$ on a Polarstar Optima plate reader (BMG, Cary, NC). Data points represent the mean of the duplicate values minus the mean of the background.

Cytokine detection. Cytokine-specific ELISAs were performed on clarified lung supernatants using DuoSet kits from R\&D Systems (Minneapolis, MN). Levels of IL-4, IL-10, IL-13, IFN- $\gamma$, TNF- $\alpha$, MIP- $1 \alpha$, and MIP- $1 \beta$ were measured using the kit protocol. Concentrations of cytokines in the lung were calculated by linear regression and expressed as $\mathrm{pg} \mathrm{ml}^{-1}$.

Measurement of luciferase expression in vivo. Mice were inoculated IVag or IM as described above with $2 \times 10^{7}$ IU of HPV PsV encapsidating a plasmid expressing the bioluminescent gene, firefly luciferase (Luc) (Promega, Madison, WI $)^{30}$ or $50 \mu \mathrm{g}$ of plasmid DNA-Luc. Empty vector-treated animals received equivalent amounts of an RFP-expressing plasmid, as naked DNA or encapsidated within HPV16. After IM injection in quadriceps, luciferase expression was measured on days $1,2,3,4,5,7$, and 14 using the IVIS 100 (Caliper Life Sciences, Hopkinton, MA). Mice were imaged before the application of substrate in order to determine whether there was any residual background from the previous day's administration. $D$-Luciferin Potassium Salt $\left(15 \mathrm{mg} \mathrm{ml}^{-1}\right.$, Caliper Life Sciences, Hopkinton MA) diluted in DMEM was delivered IVag $(20 \mu \mathrm{l})$ or IM $(50 \mu \mathrm{l})$. Three minutes post-administration of substrate, images were captured-atmedium binning with a 60-s exposure. Standardized regions of interest were created around the positive signal and photons were measured using Living Image 3.0 software. Data are reported as average radiance and plots were generated using GraphPad Prism version 5.0.

Microscopic analysis. Mice were inoculated IVag with HPV16 PsVencapsidated RFP-expressing plasmid DNA or $50 \mu \mathrm{g}$ of naked DNA (pCIR). After 2 days, reproductive tracts were harvested, frozen, and sectioned as previously described. ${ }^{29}$

Statistics. Flow cytometry, cytokine, and antibody data were analyzed by one-way statistical analysis of variance. Pair-wise comparisons were made using the Holm-Sidak, Dunn's method, or Student's $t$-test. All statistical tests were performed using SigmaStat 3.0 for Windows (Systat Software, San Jose, CA; http://www.spss.com/sigmastat).

\section{ACKNOWLEDGMENTS}

This work was supported by intramural funding from the National Institute of Allergy and Infectious Diseases and the National Cancer Institute.

\section{DISCLOSURE}

The authors have submitted a patent application related to the use of HPV PsV-encapsidated plasmids, but otherwise have no conflict of interest that would be influenced by the publication of this work.

(C) 2010 Society for Mucosal Immunology

\section{REFERENCES}

1. Ledgerwood, J.E. \& Graham, B.S. DNA vaccines: a safe and efficient platform technology for responding to emerging infectious diseases [commentary]. Hum. Vaccines 5, 623-626 (2009).

2. Brown, Z.A., Wald, A., Morrow, R.A., Selke, S., Zeh, J. \& Corey, L. Effect of serologic status and cesarean delivery on transmission rates of herpes simplex virus from mother to infant. JAMA 289, 203-209 (2003).

3. Groothuis, J.R. et al. Prophylactic administration of respiratory syncytial virus immune globulin to high-risk infants and young children. The 
Respiratory Syncytial Virus Immune Globulin Study Group. N. Engl. J. Med. 329, 1524-1530 (1993).

4. Mascola, J.R. et al. Protection of macaques against vaginal transmission of a pathogenic HIV-1/SIV chimeric virus by passive infusion of neutralizing antibodies. Nat. Med. 6, 207-210 (2000).

5. Van Kampen, K.R. et al. Safety and immunogenicity of adenovirusvectored nasal and epicutaneous influenza vaccines in humans. Vaccine 23, 1029-1036 (2005).

6. Wright, P.F. et al. Comparison of systemic and mucosal delivery of 2 canarypox virus vaccines expressing either HIV-1 genes or the gene for rabies virus G protein. J. Infect. Dis. 189, 1221-1231 (2004).

7. Lowy, D.R. \& Schiller, J.T. Prophylactic human papillomavirus vaccines. J. Clin. Invest. 116, 1167-1173 (2006).

8. Kirnbauer, R., Booy, F., Cheng, N., Lowy, D.R. \& Schiller, J.T. Papillomavirus L1 major capsid protein self-assembles into virus-like particles that are highly immunogenic. Proc. Natl. Acad. Sci. USA 89, 12180-12184 (1992).

9. Hagensee, M.E., Yaegashi, N. \& Galloway, D.A. Self-assembly of human papillomavirus type 1 capsids by expression of the $L 1$ protein alone or by coexpression of the L1 and L2 capsid proteins. J. Virol. 67, 315-322 (1993)

10. Rose, R.C., Bonnez, W., Reichman, R.C. \& Garcea, R.L. Expression of human papillomavirus type $11 \mathrm{~L} 1$ protein in insect cells: in vivo and in vitro assembly of virus like particles. J. Virol. 67, 1936-1944 (1993).

11. Kirnbauer, R. et al. Efficient self-assembly of human papillomavirus type 16 L1 and L1-L2 into virus-like particles. J. Virol. 67, 6929-6936 (1993).

12. Bontkes, H.J., Ruizendaal, J.J., Kramer, D., Meijer, C.J. \& Hooijberg, E. Plasmacytoid dendritic cells are present in cervical carcinoma and become activated by human papillomavirus type 16 virus-like particles. Gynecol. Oncol. 96, 897-901 (2005).

13. Lenz, P. et al. Papillomavirus-like particles induce acute activation of dendritic cells. J. Immunol. 166, 5346-5355 (2001).

14. Rudolf, M.P., Fausch, S.C., Da Silva, D.M. \& Kast, W.M. Human dendritic cells are activated by chimeric human papillomavirus type- 16 virus-like particles and induce epitope-specific human T cell responses in vitro. J. Immunol. 166, 5917-5924 (2001).

15. Pinto, L.A. et al. HPV-16 L1 VLP vaccine elicits a broad-spectrum of cytokine responses in whole blood. Vaccine 23, 3555-3564 (2005).

16. Harper, D.M. et al. Efficacy of a bivalent L1 virus-like particle vaccine in prevention of infection with human papillomavirus types 16 and 18 in young women: a randomised controlled trial. Lancet 364, 1757-1765 (2004).

17. Villa, L.L. et al. Prophylactic quadrivalent human papillomavirus (types 6 , $11,16$, and 18$) \mathrm{L} 1$ virus-like particle vaccine in young women: $a$ randomised double-blind placebo-controlled multicentre phase II efficacy trial. Lancet Oncol. 6, 271-278 (2005).

18. Roden, R.B. et al. In vitro generation and type-specific neutralization of a human papillomavirus type 16 virion pseudotype. J. Virol. 70, 5875-5883 (1996).

19. Unckell, F., Streeck, R.E. \& Sapp, M. Generation and neutralization of pseudovirions of human papillomavirus type 33. J. Virol. 71, 2934-2939 (1997).

20. Kawana, K., Yoshikawa, H., Taketani, Y., Yoshiike, K. \& Kanda, T. In vitro construction of pseudovirions of human papillomavirus type 16: incorporation of plasmid DNA into reassembled L1/L2 capsids. J. Virol. 72, 10298-10300 (1998).

21. Touze, A. \& Coursaget, P. In vitro gene transfer using human papillomavirus-like particles. Nucleic Acids Res. 26, 1317-1323 (1998).

22. Combita, A.L., Touze, A., Bousarghin, L., Sizaret, P.Y., Munoz, N. \& Coursaget, P. Gene transfer using human papillomavirus pseudovirions varies according to virus genotype and requires cell surface heparan sulfate. FEMS Microbiol. Lett. 204, 183-188 (2001).

23. Buck, C.B., Pastrana, D.V., Lowy, D.R. \& Schiller, J.T. Efficient intracellular assembly of papillomaviral vectors. J. Virol. 78, 751-757 (2004).

24. Buck, C.B., Thompson, C.D., Pang, Y.Y., Lowy, D.R. \& Schiller, J.T. Maturation of papillomavirus capsids. J. Virol. 79, 2839-2846 (2005).

25. Huang, Y., Fayad, R., Smock, A., Ullrich, A.M. \& Qiao, L. Induction of mucosal and systemic immune responses against human carcinoembryonic antigen by an oral vaccine. Cancer Res. 65, 6990-6999 (2005).

26. Shi, W., Liu, J., Huang, Y. \& Qiao, L. Papillomavirus pseudovirus: a novel vaccine to induce mucosal and systemic cytotoxic T-lymphocyte responses. J. Virol. 75, 10139-10148 (2001).

27. Zhang, H., Fayad, R., Wang, X., Quinn, D. \& Qiao, L. Human immunodeficiency virus type 1 gag-specific mucosal immunity after oral immunization with papillomavirus pseudoviruses encoding gag. J. Virol. 78, 10249-10257 (2004).

28. Zhang, H., Huang, Y., Fayad, R., Spear, G.T. \& Qiao, L. Induction of mucosal and systemic neutralizing antibodies against human immunodeficiency virus type 1 (HIV-1) by oral immunization with bovine Papillomavirus-HIV-1 gp41 chimeric virus-like particles. J. Virol. 78, 8342-8348 (2004).

29. Roberts, J.N. et al. Genital transmission of HPV in a mouse model is potentiated by nonoxynol-9 and inhibited by carrageenan. Nat. Med. 13, 857-861 (2007).

30. Johnson, K.M., Kines, R.C., Roberts, J.N., Lowy, D.R., Schiller, J.T. \& Day, P.M. Role of heparan sulfate in attachment to and infection of the murine female genital tract by human papillomavirus. J. Virol. 83, 2067-2074 (2009).

31. Ruckwardt, T.J., Bonaparte, K.L., Nason, M.C. \& Graham, B.S. Regulatory $T$ cells promote early influx of CD8+ $T$ cells in the lungs of respiratory syncytial virus-infected mice and diminish immunodominance disparities. J. Virol. 83, 3019-3028 (2009).

32. Graham, B.S. \& Walker, C. Meeting the challenge of vaccine design to control HIV and other difficult viruses. In Immunology of Infectious Diseases. (Kaufmann, S.H.E., Rouse, B., \& Sacks, D., eds) (Washington DC, ASM Press, 2010).

33. Lubeck, M.D. et al. Long-term protection of chimpanzees against high-dose HIV-1 challenge induced by immunization. Nat. Med. 3, 651-658 (1997).

34. Andino, R. et al. Engineering poliovirus as a vaccine vector for the expression of diverse antigens. Science 265, 1448-1451 (1994).

35. Arnold, G.F., Resnick, D.A., Smith, A.D., Geisler, S.C., Holmes, A.K. \& Arnold, E. Chimeric rhinoviruses as tools for vaccine development and characterization of protein epitopes. Intervirology 39, 72-78 (1996).

36. Buchholz, U.J., Granzow, H., Schuldt, K., Whitehead, S.S., Murphy, B.R. \& Collins, P.L. Chimeric bovine respiratory syncytial virus with glycoprotein gene substitutions from human respiratory syncytial virus (HRSV): effects on host range and evaluation as a live-attenuated HRSV vaccine. J. Virol. 74, 1187-1199 (2000).

37. Bukreyev, A. et al. Recombinant Newcastle disease virus expressing a foreign viral antigen is attenuated and highly immunogenic in primates. J. Virol. 79, 13275-13284 (2005).

38. Widera, G. et al. Increased DNA vaccine delivery and immunogenicity by electroporation in vivo. J. Immunol. 164, 4635-4640 (2000).

39. Geiben-Lynn, R., Greenland, J.R., Frimpong-Boateng, K. \& Letvin, N.L. Kinetics of recombinant adenovirus type 5 , vaccinia virus, modified vaccinia ankara virus, and DNA antigen expression in vivo and the induction of memory T-lymphocyte responses. Clin. Vaccine Immunol. 15, 691-696 (2008).

40. Tang, Y.W. \& Graham, B.S. Anti-IL-4 treatment at immunization modulates cytokine expression, reduces illness, and increases cytotoxic $T$ lymphocyte activity in mice challenged with respiratory syncytial virus. J. Clin. Invest. 94, 1953-1958 (1994).

41. Aung, S., Tang, Y.W. \& Graham, B.S. Interleukin-4 diminishes CD8(+) respiratory syncytial virus-specific cytotoxic T-lymphocyte activity in vivo. J. Virol. 73, 8944-8949 (1999).

42. Delgado, M.F. et al. Lack of antibody affinity maturation due to poor Toll-like receptor stimulation leads to enhanced respiratory syncytial virus disease. Nat. Med. 15, 34-41 (2009).

43. Mesquita, P.M. et al. Disruption of tight junctions by cellulose sulfate facilitates HIV infection: model of microbicide safety. J. Infect. Dis. 200, 599-608 (2009).

44. Barouch, D.H. et al. A human T-cell leukemia virus type 1 regulatory element enhances the immunogenicity of human immunodeficiency virus type 1 DNA vaccines in mice and nonhuman primates. J. Virol. 79, 8828-8834 (2005).

45. Buck, C.B. \& Thompson, C.D. Production of papillomavirus-based gene transfer vectors. Curr. Protoc. Cell. Biol. Supplement 37, Unit 261 (2007).

46. Dalal, S.J., Estep, J.S., Valentin-Bon, I.E. \& Jerse, A.E. Standardization of the Whitten Effect to induce susceptibility to Neisseria gonorrhoeae in female mice. Contemp. Top Lab. Anim. Sci. 40, 13-17 (2001).

47. Graham, B.S., Perkins, M.D., Wright, P.F. \& Karzon, D.T. Primary respiratory syncytial virus infection in mice. J. Med. Virol. 26, 153-162 (1988).

48. Johnson, T.R., Johnson, J.E., Roberts, S.R., Wertz, G.W., Parker, R.A. \& Graham, B.S. Priming with secreted glycoprotein G of respiratory syncytial virus (RSV) augments interleukin-5 production and tissue eosinophilia after RSV challenge. J. Virol. 72, 2871-2880 (1998).

49. Rutigliano, J.A., Ruckwardt, T.J., Martin, J.E. \& Graham, B.S. Relative dominance of epitope-specific CD8+ T cell responses in an F1 hybrid mouse model of respiratory syncytial virus infection. Virology 362, 314-319 (2007).

50. Kirnbauer, R. et al. Virus-like particles of bovine papillomavirus type 4 in prophylactic and therapeutic immunization. Virology 219, 37-44 (1996). 